\title{
Adequacy of fluid balance chart documentation on wards
}

\author{
Authors: Aliya Nazli, Frederique Brigham-Chan, Melvin Fernandes and Aziz Anjum
}

\section{Aims}

To to ensure that fluid balance charts are completed adequately for clinicians to compose safe management plans for patients.

\section{Methods}

This is a retrospective audit against a standard adapted from an NHS trust guideline. The standards audited against were (1) oral fluids to be documented in $\mathrm{mL}$, (2) IV fluids to be documented in $\mathrm{mL}$, (3) fluid output to be documented in $\mathrm{mL}$, and (4) documentation of receptacle emptying. Data collection involved review of fluid balance charts on two surgical wards over five randomly chosen days in December 2014 and data were analysed on MS Excel.

\section{Results}

The audit found an overall of $52 \%$ of fluid balance charts that were inadequately completed. Oral and IV fluid input were $94 \%$ and $98 \%$ compliant respectively, with only $50 \%$ compliance in fluid output. Recording whether receptacles were emptied was done particularly poorly, with $23 \%$ compliance to the standard. The audit provided the following recommendations for quality improvement, all of which were implemented: (1) educate staff about the importance of fluid balance recordkeeping in patient care, (2) provide accessible guidelines for staff to use on the wards, (3) re-audit.

The re-audit involved the same method. Following the audit recommendations, the re-audit results showed a remarkable improvement, with inadequately completed charts dropping to $38 \%$. Fluid input and output records were $100 \%$ and $62 \%$ compliant respectively, with an improvement to the recording of receptacle emptying at $64 \%$.

\section{Conclusions}

Standardised and unambiguous documentation of fluid balance is a key part of managing patients. The education and training of both doctors and nurses, along with clear guidelines, can greatly improve the documentation and management of fluid balance. Hence, it is vital that staff involved in the care of patients understand the reasons why recording fluid balance is important, thereby maintaining a high standard of care as well as patient safety. 